Cite this: RSC Adv., 2014, 4, 15293

\title{
Hydrophobic coordination polymer nanoparticles and application for oil-water separation $\dagger$
}

\author{
F. Novio ${ }^{a b}$ and D. Ruiz-Molina*ab
}

Received 29th January 2014

Accepted 14th March 2014

DOI: $10.1039 / c 4 r a 00850 b$

www.rsc.org/advances

Catechol chemistry is used to fabricate coordination polymer nanoparticles bearing long alkyl chains with enhanced thermal and colloidal stabilities and applications for hydrophobic surfaces and oil-water separation.

Having control over the chemistry of colloid surfaces has attracted the interest of scientists for many years as they play a critical role in many chemical and life science processes; by chemically modifying the surface or by adding other components one can enhance their stability and performance. ${ }^{1}$ Over the past decades, different experimental approaches have been developed with this aim and a vast amount of basic knowledge has been accumulated in nanoparticles, becoming a battleship for many chemists. One of the latest families to be incorporated is that of amorphous coordination polymer particles (CPPs). ${ }^{2}$ Despite being first described less than a decade ago, ${ }^{3}$ CPPs (combining metal ions and organic ligands) have allowed for new amazing functions/properties ${ }^{4}$ and applications. ${ }^{5}$ Rapid innovations in all these fields have increased the likelihood that CPPs will eventually come into several other materials and media. Therefore, the development of new approaches to enhance their processability represents a challenge, among them, the fabrication of hydrophobic CPPs.

The most direct approach to achieve this goal is a priori the hierarchical assembly of nanoparticles using ligands with long alkyl chains, a small fraction of which is expected to remain on the nanoparticle surface. With this aim, we fabricated hydrophobic nanoparticles with the general composition [Co(bix)(hdcat $)_{2}$, where bix is the ligand 1,4-bis(imidazol-1-ylmethyl)

${ }^{a}$ ICN2 - Institut Catala de Nanociencia i Nanotecnologia, Campus UAB, 08193 Bellaterra (Barcelona), Spain. E-mail: druiz@cin2.es

${ }^{b}$ CSIC - Consejo Superior de Investigaciones Cientificas, ICN2 Building, Campus UAB, 08193 Bellaterra (Barcelona), Spain

$\dagger$ Electronic supplementary information (ESI) available: Methods, FT-IR/UV-Vis/XPS characterization, study of colloidal stability, BET characterization, CPP4 characterization, nanoparticles thermal characterization. See DOI: $10.1039 / \mathrm{c} 4 \mathrm{ra} 00850 \mathrm{~b}$ benzene, a flexible bisimidazole bridging ligand used to induce polymerization, and hdcat is the ligand 4-heptadecylcatechol bearing a long alkyl chain. A schematic representation of this approach is shown in Fig. 1. The choice of this selection is justified by: (1) the high affinity of catechol groups to coordinate to different metal ions, provides us with a simple way to incorporate the alkyl chain to the polymer backbone; ${ }^{6}(2)$ the valence tautomerism exhibited by [Co(bix)(hdsq)(hdcat)] can be exploited to assess morphological modifications ${ }^{7}$ and (3) mussel-inspired catechols have already been successfully used to fabricate efficient hydrophobic coatings and oil-water separation materials. ${ }^{8}$

In a typical experiment bix and hdcat were firstly dissolved in ethanol and treated with an aqueous solution of $\mathrm{Co}\left(\mathrm{CH}_{3-}\right.$ $\mathrm{COO})_{2} \cdot 4 \mathrm{H}_{2} \mathrm{O}$. After vigorous stirring at room temperature for 30 minutes the solution turned light brown and led to the rapid formation of a precipitate that was collected by centrifugation

a<smiles>CCCCCCCCCCCCCCCCCc1ccc(O)c(O)c1</smiles>

b

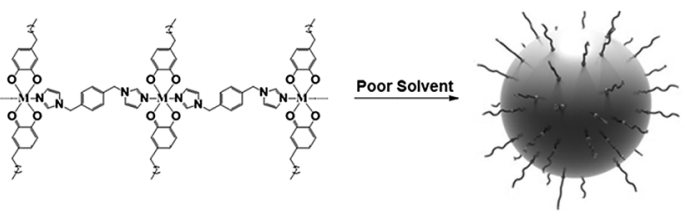

C

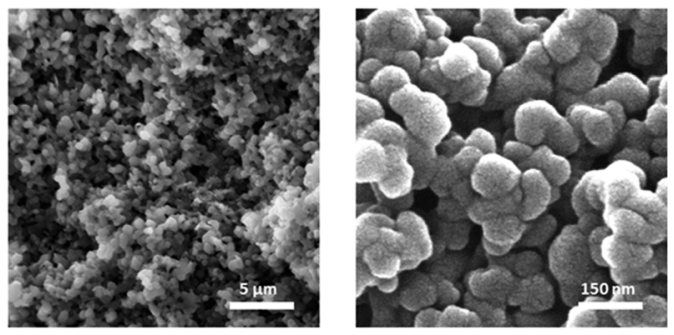

Fig. 1 (a) Schematic of 4-heptadecylcatechol (hdcat) ligand. (b) Schematic of one-pot synthesis of CPP1 hydrophobic nanoparticles. (b) SEM images of CPP1 nanoparticles at different magnifications. 
and washed with ethanol several times (for experimental details see ESI, $\dagger$ S1). As can be seen in Fig. 1c, SEM images revealed the formation of nanoparticles (CPP1) with an average diameter of $115 \pm 21 \mathrm{~nm}$. X-ray powder diffraction data showed the typical pattern for an amorphous material. Infrared spectra (FT-IR) evidenced the coordination of the bix and hdcat ligands to the metal, UV-Vis spectra showed the typical electronic transitions of these cobalt polymeric compounds, ${ }^{7}$ and X-ray photoelectron spectroscopy (XPS), which showed the presence of chemically bound catechol to cobalt metal ions (see ESI, $\uparrow \mathrm{S} 2-\mathrm{S} 4$ ).

Dispersion of CPP1 nanoparticles in ethanol results in the formation of stable colloidal suspensions with tendency to float and aggregate in water showing its high hydrophobic character. On the contrary, in non-polar solvents such as hexane nanoparticles are totally soluble; SEM images of the resulting materials after solvent evaporation confirm the formation of a non-structured amorphous material lacking original nanoparticles. In fact, if the solution is maintained on air for a few days decomposition of the polymer takes place most likely due to oxidation of the catechol units upon prolonged contact with the atmospheric oxygen (see ESI, $\uparrow$ S5).

An alternative approach to obtain stable colloidal dispersions of CPPs on organic solvents is the postsynthetic functionalization, as already reported for of metal-organic frameworks ${ }^{9}$ and for amorphous coordination polymer particles. ${ }^{10}$ For this, the two step-methodology shown in Fig. 2a is used. First, robust hydrophilic nanoparticles (CPP2) reinforced by several hydrogen bonds within the polymeric network were obtained by using a catechol ligand bearing a carboxylic group. Afterwards, the surface carboxylates were functionalized with a long alkyl chain (octadecylamine) through a well-known condensation reaction using the standard coupling reagents 1-ethyl-3-(3-dimethylaminopropyl)carbodiimide (EDC) and
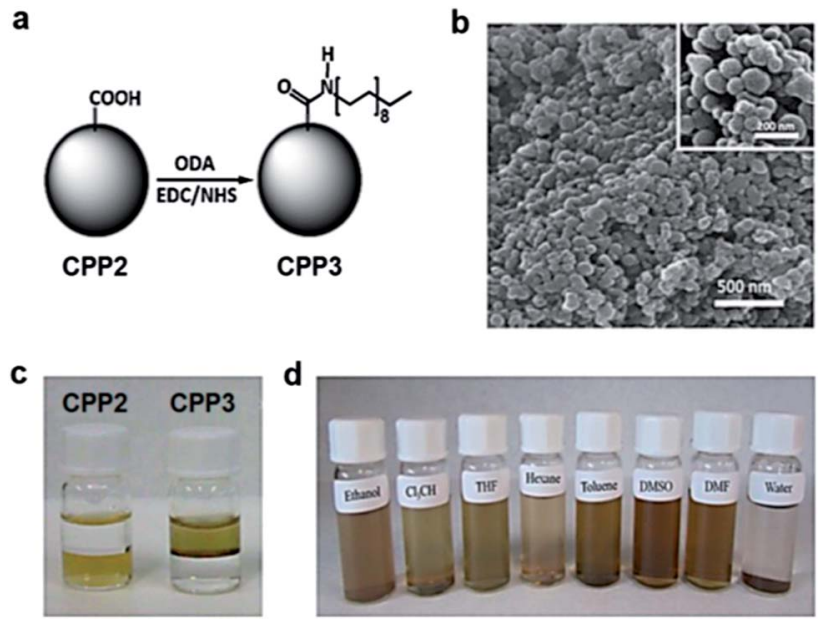

Fig. 2 Colloidal stability of CPPs in organic media. (a) Schematic of the coupling reaction between nanoparticles containing surface carboxyl groups and octadecylamine chains. (b) SEM image of CPP3 nanoparticles (inset: zoom). (c) Separation in a biphasic mixture toluene (top)-water (down) of the hydrophilic CPP2 and the hydrophobic CPP3 nanoparticles. (d) Dispersion of CPP3 in different organic solvents and water.
$\mathrm{N}$-hydroxysuccinimide (NHS) (for synthetic details see Methods section in ESI, $\uparrow$ S1). TEM and SEM images revealed that the resulting nanoparticles (CPP3) maintain the spherical shape while being reasonably monodisperse, with average diameters of $112 \pm 12 \mathrm{~nm}$, a value slightly higher than that for the initial CPP2 nanoparticles $(110 \pm 17 \mathrm{~nm})$. The FT-IR spectra confirmed coupling by the presence of bands associated to the alkyl group (see ESI, $\uparrow \mathrm{S} 2$ ). BET measurements leaded to small specific areas with small differences between the synthesized CPPs (see ESI, $\uparrow$ S6). Although the determination of the accessible carboxyl groups on the surface to react with amine groups is difficult, in a recent work an approximated value of $1.5-2.0 \times 10^{-9} \mathrm{~mol}$ $\mathrm{mg}^{-1}$ was obtained by reaction of CPP2 nanoparticles with a primary amine label fluorophore and subsequent spectroscopic measurements. ${ }^{10}$

Interestingly, in this case, the hydrophobic shell given by the octadecylamine groups over the robust carboxyl-functionalized core allowed for a very good colloidal dispersion in different organic solvents such as ethanol, chloroform, hexane, toluene, THF, DMSO and DMF. However, CPP3 nanoparticles precipitate in water (see Fig. 2d). Likewise, a biphasic mixture of toluene (upper phase)-water (bottom phase) reveals that hydrophilic CPP2 nanoparticles remain on the aqueous phase whereas CPP3 stay in the organic solvent due to their different wettability characteristics (see Fig. 2c and d). In addition to dissimilarities in the colloidal stability, significant different thermal behaviour was found for nanoparticle CPP1-CPP3. Combined TGA/DSC and SEM analysis showed that CPP1 nanoparticles began to melt at only 65 to $70{ }^{\circ} \mathrm{C}$ and decompose just above $100{ }^{\circ} \mathrm{C}$, whereas CPP3 remained stable at least up to $175{ }^{\circ} \mathrm{C}$. SEM images of the morphological evolution of both families of nanoparticles at three different temperatures are shown in
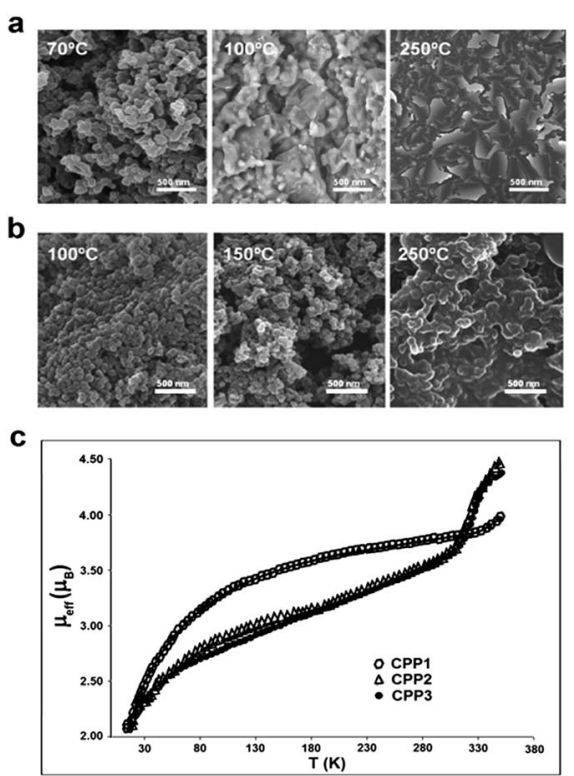

Fig. 3 Morphological variation of (a) CPP1 and (b) CPP3 nanoparticles at three specific temperatures. (c) $\mu_{\text {eff }}$ values as a function of temperature for the amorphous nanoparticles of CPP1, CPP2 and CPP3. 
Fig. 3 for comparison purposes (TGA/DSC are shown in the $\mathrm{ESI}, \dagger \mathrm{S} 7)$. The presence of the long alkyl chains not only on the surface but all over the CPP1 nanoparticles induces a decrease of the cohesive forces with respect to the hydrogen-bonded stabilized CPP3 nanoparticles, and therefore, a decrease of its thermal stability.

The amorphous nature of CPP1-3 nanoparticles precludes any accurate structural characterisation by classical diffraction techniques. Nevertheless, we exploited the valence tautomerism (VT) behaviour shown by the family of [Co(bix)(sq)(cat)] nanoparticles to investigate their structural similarities. ${ }^{7}$ These systems might interconvert reversibly between the low-spin ls-[Co $\left.{ }^{\mathrm{III}}(\mathrm{bix})(\mathrm{sq})(\mathrm{cat})\right]$ and high-spin hs-[Co $\left.{ }^{\mathrm{II}}(\mathrm{bix})(\mathrm{sq})_{2}\right]$ tautomers, where sq and cat stand respectively for the semiquinone and catecholate form, by an intramolecular metal-ligand electron transfer. ${ }^{11}$ Moreover, VT can be selectively monitored by the temperature-dependent magnetization measurements shown in Fig. $3 \mathrm{~b}$.

As can be seen there, all the different samples studied exhibit temperature-dependence of their effective magnetic moment $\left(\mu_{\text {eff }}\right)$ consistent with the presence of a VT interconversion from low- to high-spin states for a large fraction of molecules. Two main conclusions can be extracted from there. First, the occurrence of VT corroborates the expected [Co(bix)(sq)(cat)] composition. And second, it has already been shown that the profile of $\mu_{\text {eff }} v s$. $T$ plot is highly sensitive not only to the chemical structure but also to the local environment. In other words, complexes containing similar chemical structure may exhibit or not VT, or the low-spin-to-high-spin conversion might take place at different temperatures depending on structural and environmental parameters. This fact can be the origin for the divergent magnetic behaviour found for CPP1 and CPP3 nanoparticles according to their different composition. The interplay of supramolecular interactions between ligands clearly modifies the cohesiveness of the nanoparticles and therefore the resulting bulk material properties according to the differential chemical nature of the groups, non-specific cohesive forces and/or steric constraints. Worth to mention, no significant differences are found between particles CPP2 and CPP3 where only the surface has been functionalized confirming that the surface functionalization does not alter or modifies the nature of the nanoparticles.

As an example for applications, CPP3 nanoparticles were used for separating oils from water. By simply placing the powder material on the surface of oil-water mixtures, we were able to quickly and selectively remove oils from the water in a few minutes. Interestingly, the CPP3 nanoparticles with the adsorbed oil are retained upon filtration leading to clean water as shown in Fig. $4 \mathrm{~b}$.

The surface wettability of glass substrates coated with our CPP nanoparticles were also characterized by the water contact angle measurement (see Fig. 4c). While the bare glass and that coated with CPP2 nanoparticles displays a rather hydrophilic character, glass substrates coated with CPP1 and CPP3 show contact angles of 118 and $97^{\circ} \mathrm{C}$, respectively.

Finally, we also envisioned the fabrication of hydrophobic CPP platforms bearing iron metal ions (CPP4) following the same two-step methodology. TEM and SEM images revealed
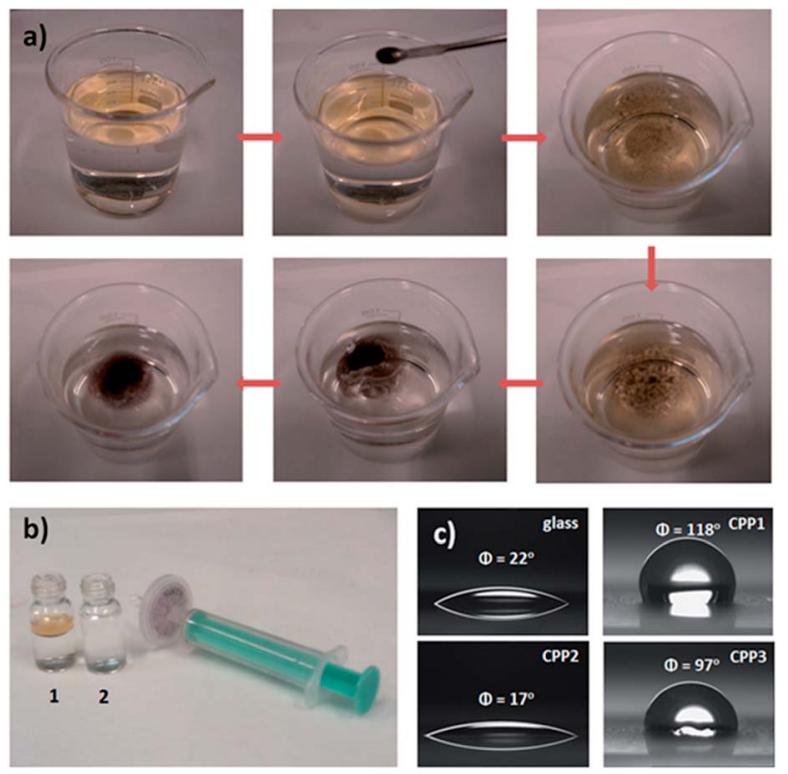

Fig. 4 (a) Collection of oil (n-tetradecane) from water surface by hydrophobic nanoparticles (CPP3). (b) Filtration of non-treated oilwater mixture (1) and treated mixture with hydrophobic nanoparticles (2); the hydrophobic nanoparticles retains the oil in the syringe filter. (c) Contact angle of an untreated glass and treated glass with CPP1, CPP2 and CPP3 nanoparticles.

that the resulting nanoparticles maintain the spherical shape with average diameters of $98 \pm 8 \mathrm{~nm}$ (see ESI, $\uparrow$ S8) and exhibit very similar wettability properties to those shown by CPP3 nanoparticles. This result confirms the potential of our new synthetic framework to be extrapolated to other metal ions with different functionalities.

In summary, two new families of catechol-based hydrophobic CPP nanoparticles with different properties have been reported. The first approach consist of a one-pot reaction where the hierarchical assembly of nanoparticles takes place using the 4-heptadecylcatechol, a small fraction of which remains on the nanoparticle surface. The second approach consists of a two-step methodology where the use of ligands bearing a carboxylic group enables reproducible fabrication of nanoscale platforms and their post-synthetic modification with octadecylamine. Most significant divergences between both families of nanoparticles come from their different thermal and colloidal stability. Combined TGA/DSC analysis showed that CPP1 nanoparticles are thermally less stable. Moreover, CPP3 nanoparticles formed stable colloidal suspensions in several different organic solvents according to their hydrophilic/hydrophobic core-shell character whereas CPP1 nanoparticles were totally soluble in hexane. Magnetization measurements also indicate that both families of CPPs must be formed by equivalent coordination polymers in rather different phases or environments. So, with a clever choice and combination of core materials new possibilities and applications for hydrophobic nanoparticles with tuneable characteristics could be brought up.

The hydrophobic nanoparticles can also act as sponges effectively separating oils from water surface trough a simple filtration process while conferring hydrophobic character to 
surface upon deposition. With an elaborate choice of precursors by taking advantage of coordination chemistry, multifunctional CPPs systems might be fabricated for self-cleaning, environmental remediation and so on. Moreover, the results obtained should provide useful insights into the factors that affect the formation of nanostructured coordination polymers and the resulting bulk properties. Conformational modulation under the influence of long alkyl chains or carboxylic coligands with different supramolecular interactions and constraints interactions has been demonstrated. More systematic studies are needed though to control and understand particle morphology. ${ }^{12}$

\section{Acknowledgements}

We acknowledge the financial support of the Ministerio de Economía y Competitividad through project MAT2012-38318C03-02. F.N. thanks the Ministerio de Economía y Competitividad (MINECO) for a JdC (JCI-2011-09239) post-doctoral grant. Thanks to COST Action MP1106.

\section{Notes and references}

1 R. A. Sperling and W. J. Parak, Philos. Trans. R. Soc., A, 2010, 368, 1333.

2 S. R. Batten, N. R. Champness, X. M. Chen, J. GarciaMartinez, S. Kitagawa, L. Öhrström, M. O'Keeffe, M. P. Suhh and J. Reedijk, CrystEngComm, 2012, 14, 3001.

3 (a) M. Oh and C. A. Mirkin, Nature, 2005, 438, 651; (b) X. Sun, S. Dong and E. Wang, J. Am. Chem. Soc., 2005, 127, 13102.

4 (a) F. Novio, J. Simmchen, N. Vázquez, L. Amorín and D. Ruiz-Molina, Coord. Chem. Rev., 2013, 257, 2839; (b) J. Della Rocca, D. Liu and W. Lin, Acc. Chem. Res., 2011, 44, 957; (c) X. Wang and R. McHale, Macromol. Rapid Commun., 2010, 31, 331; (d) W. Lin, W. J. Rieter and K. M. Taylor, Angew. Chem., Int. Ed., 2009, 48, 650; (e) A. Spokoyny, D. Kim, A. Sumrein and C. A. Mirkin, Chem. Soc. Rev., 2009, 38, 1218; (f) V. A. Friese and D. G. Kurth, Coord. Chem. Rev., 2008, 252, 199.

5 (a) T. Q. Hung, F. Terki, S. Kamara, M. Dehbaoui, S. Charar, B. Sinha, C. Kim, Ph. Gandit, I. Gural'skiy, G. Molnar, L. Salmon, H. J. Shepherd and A. Bousseksou, Angew.
Chem., Int. Ed., 2013, 52, 1185; (b) L. Xing, H. Zheng, Y. Cao and S. Che, Adv. Mater., 2012, 24, 6433; (c) R. C. Huxford, K. E. deKrafft, W. S. Boyle, D. Liu and W. Lin, Chem. Sci., 2012, 3, 198; (d) H. J. Lee, K. Lee, W. Cho, Y. J. Cho and M. Oh, CrystEngComm, 2012, 14, 2837; (e) F. Prins, M. Monrabal-Capilla, E. A. Osorio, E. Coronado and H. S. J. van der Zant, Adv. Mater., 2011, 23, 1545; $(f)$ I. Imaz, M. Rubio-Martínez, L. GarcíaFernández, F. García, D. Ruiz-Molina, J. Hernando, V. Puntes and D. Maspoch, Chem. Commun., 2010, 46, 4737; $(g)$ C. Aimé, R. Nishiyabu, R. Gondo and N. Kimizuka, Chem.-Eur. J., 2010, 16, 3604; (h) W. J. Rieter, K. M. Pott, M. L. Taylor and W. Lin, J. Am. Chem. Soc., 2008, 130, 11584; (i) X. Roy, J. K.-H. Hui, M. Rabnawaz, G. Liu and M. J. MacLachlan, Angew. Chem., Int. Ed., 2011, 50, 1597.

6 I. Imaz, J. Hernando, D. Ruiz-Molina and D. Maspoch, Angew. Chem., Int. Ed., 2009, 48, 2325.

7 L. Amorín-Ferré, F. Busqué, J. L. Bourdelande, D. RuizMolina, J. Hernando and F. Novio, Chem.-Eur. J., 2013, 19, 17508.

8 (a) J. Saiz-Poseu, J. Sedó, B. García, T. Parella, R. Alibés, J. Hernando, F. Busqué and D. Ruiz-Molina, Adv. Mater., 2013, 25, 2066; (b) Y. Cao, X. Zhang, L. Tao, K. Li, Z. Xue, L. Feng and Y. Wei, ACS Appl. Mater. Interfaces, 2013, 5, 4438; (c) Q. Zhu and Q. Pan, ACS Nano, 2014, 8, 1402; (d) A. I. Neto, H. J. Meredith, C. L. Jenkins, J. J. Wilker and J. F. Mano, RSC Adv., 2013, 3, 93529356.

9 (a) K. M. L. Taylor-Pashow, J. D. Rocca, Z. Xie, S. Tran and W. Lin, J. Am. Chem. Soc., 2009, 14, 14261; (b) S. M. Cohen, Chem. Rev., 2012, 112, 970.

10 F. Novio, J. Lorenzo, F. Nador, K. Wnuk and D. Ruiz-Molina, Adv. Healthcare Mater., submitted.

11 (a) D. N. Hendrickson and C. G. Pierpont, Top. Curr. Chem., 2004, 234; (b) P. Gutlich and A. Dei, Angew. Chem., Int. Ed. Engl., 1997, 36, 2734; (c) E. Evangelio and D. Ruiz-Molina, Eur. J. Inorg. Chem., 2005, 2957.

12 V. J. Richards, A. J. Blake, W. Lewis, H. Nowell, C. D. J. Parmenter and N. R. Champness, CrystEngComm, 2013, 15, 9704. 